A.S. Kuznetsov, M.A. Gleeson, F. Bijkerk,

Thin Solid Films, 545, 571-579, (2013),

http://dx.doi.org/10.1016/j.tsf.2013.07.039

\title{
Hydrogen-induced blistering of Mo/Si multilayers: uptake and distribution
}

\author{
A. S. Kuznetsova, M. A. Gleesona,, F. Bijkerka,b. \\ a FOM Institute DIFFER - Dutch Institute for Fundamental Energy Research, Postbus 1207, 3430 BE Nienvegein, \\ The Netherlands. (www.differ.nl) \\ b MESA+ Institute for Nanotechnology, University of Twente, Postbus 217, 7500 AE Enschede, The Netherlands.
}

\begin{abstract}
We report on the uptake of deuterium by thin-film $\mathrm{Mo} / \mathrm{Si}$ multilayer samples as a result of exposure to fluxes of predominantly thermal atomic and molecular species, but also containing a small fraction of energetic (800-1000 eV) ions. These exposures result in blister formation characterized by layer detachment occurring exclusively in the vicinity of the Mo-on-Si interfaces. This localization is attributed to strained centres introduced within the interfacial region during silicide formation and subsequent Mo crystallization. The correlation between D-content and blistering was studied. After an initial uptake period the D-content stabilized at $\sim 1.3 \times 10^{16}$ at. $/ \mathrm{cm}^{2}$. Blister development is not simply a function of the content. Different blistering processes are simultaneously operative, with three distinct distributions being observed. The areal number densities of the initial two blister distributions to appear are established before the content stabilizes, while the multilayer is susceptible to successive stages of blistering associated with the effects of energetic ions. H-atom depth profiling of hydrogen-exposed samples by resonant nuclear reaction analysis shows preferential accumulation in the Mo layers. A distinct local maximum with a remarkably high hydrogen concentration $(\sim 19$ at.\%) develops in the outermost Mo layer. This is attributed to enhanced accommodation of hydrogen in voids and vacancies within the layer as a consequence of its polycrystalline structure and its highlyconstrained state.
\end{abstract}

|Keywords: Blistering; Multilayer; Hydrogen-induced; Hydrogenation; Ion-induced; Hydrogen uptake; Hydrogen concentration profile; Nuclear reaction analysis.

\footnotetext{
*Corresponding author: e-mail: M.A.Gleeson@differ.nl
} 


\section{Introduction}

Processing and deposition of layered materials on the nano-scale level are becoming increasingly important for technological applications [1, 2]. Consequently, it is necessary to understand the properties of such materials and how they interact with, and are modified by, their processing and operational environments. The interaction of hydrogen with multilayer (ML) structures is a particular case-in-point. The highly-constrained state of the materials within such structures can dramatically affect their interaction with hydrogen [3]. Simple extrapolation for the bulk material properties cannot be assumed. Hydrogen can lead to significant modification of the optical, electronic, magnetic, and structural properties of thin films and MLs. For instance, it can be used to alter the magnetic properties of ultrathin metal MLs [4-6]. A possible negative consequence of the interaction with hydrogen is the formation of blisters. Several examples of such effects have been reported in a variety of MLs. Blistering has been observed as a result of hydrogen absorption by magnetic Co/Pd MLs [6]; blisters and craters formed during annealing of hydrogenated Si-Ge ML structures related to multi-junction solar cells technology [7]; hydrogen ion irradiation induced blistering of Mo/Si MLs designed for

solar missions [8]. It has been shown that hydrogen-induced blistering of Mo/Si MLs can have a strong dependence on the precise composition of the incident flux [9].

In order to identify the key parameters influencing the formation of blisters, an in-depth understanding of the processes involved in hydrogen interaction with ML structures is required. This will allow estimation of the level of structural modification that can be anticipated prior to exposure to specific hydrogen-containing environments and will facilitate the development of mitigation strategies to prevent blister formation. Conversely, in relation to silicon-on-insulator technologies [10, 11], enhanced localization and rate of layer detachment are desirable for thinfilm transfer processes that involve hydrogenation steps $[12,13]$. In both cases, understanding of 
the correlation between the hydrogen fluence received and the resultant hydrogen content and distribution in the layered structure is desirable.

In the current manuscript we have tracked hydrogen uptake and distribution among the outermost layers of a Mo/Si ML. Elastic recoil detection (ERD) has been used to determine the time dependence of the D-uptake by samples exposed to a deuterium flux. Atomic force microscopy (AFM) measurements were used to correlate the resultant blister formation with the D-content. Independently, resonant nuclear reaction analysis (NRA) was used to obtain $\mathrm{H}$-atom depth profiles of hydrogen-exposed samples. The H-content retained by individual layers is related to their respective structures: amorphous $\mathrm{Si}(a-\mathrm{Si})$ versus polycrystalline Mo. The development of blisters is discussed in light of existing work involving the use of buried strained layers, most notable $\mathrm{SiGe}$, in order to produce controlled delamination of thin Si overlayers [1215]. A critical factor is the introduction of localized strained states as a result of the formation of a silicide layer and subsequent Mo crystallization during deposition of Mo on a-Si. Combined, the results provide insight into the complexities of hydrogen-uptake and the associated blistering of a nano-layered structure.

\section{Experimental details}

The samples under investigation were deposited at room temperature using in-house deposition facilities. The pre-deposition pressure in this system was $<1 \times 10^{-6} \mathrm{~Pa}$, the main constituent of which was water. The samples were composed of $50 \mathrm{Mo} / \mathrm{Si}$ bilayers deposited on an ultra-smooth Si wafer by magnetron sputtering utilizing Krypton as the operating gas. The first layer deposited (directly on the Si wafer) was a Si layer and the stack was also terminated with a Si layer. The individual layer deposition times were $\sim 60$ (Si) and $\sim 70$ (Mo) seconds. Additionally, $100 \mathrm{eV} \mathrm{Kr}$ ion polishing ( $25 \mathrm{~s}$ ) using a Kaufmann ion source was applied to the as-deposited Si layers in order to minimize the development of interfacial roughness [16]. This 
ion polishing represented an "etch-back" of approximately $10 \%$ of the as-deposited layer. The interval between individual deposition/etch steps was $\sim 35 \mathrm{~s}$.

Individual layer thicknesses of $\sim 2.7 \mathrm{~nm}$ for $\mathrm{Mo}$ and $\sim 4.65 \mathrm{~nm}$ for Si were derived from Rutherford Backscattering Spectrometry (RBS) measurements. These estimates are based on bulk material densities, and non-uniformities arising from intermixing and compound formation at the interfaces are not factored in the estimations. Hydrogen exposures were performed in a separate vacuum chamber operated at a base pressure of $\sim 1 \times 10^{-6} \mathrm{~Pa}$. The samples were exposed to the flux from a thermal capillary cracker source (Oxford Applied Research TC50) operated at a power of $55 \mathrm{~W}$ using either $\mathrm{D}_{2}$ or $\mathrm{H}_{2}$ at gas flows of 2 and $1 \mathrm{sccm}$, respectively. Assuming a $50 \%$ cracking probability, the resultant neutral atomic fluxes would be on the order of $\sim 10^{17} \mathrm{~cm}$ ${ }^{2} \mathrm{~s}^{-1}$. In addition to the neutral flux, the cracker also produced an ion current on the order of $\sim 75-150 \mathrm{nA} / \mathrm{cm}^{2}$ depending on the flow rate. Thus, the ion fraction in the flux was on the order of $10^{-6}$. Although the ion energy spanned the range from $0-1000 \mathrm{eV}$, the majority of ions had an energy $>800 \mathrm{eV}$. All samples were maintained at $100{ }^{\circ} \mathrm{C}$ during exposure. Under such conditions, these samples typically exhibited pronounced blistering on a 3-5 hour timescale (neutral fluences on the order of $10^{21} \mathrm{~cm}^{-2}$ [ [9].

Samples were transported through air both before and after exposure. No special treatment was applied to the samples pre- or post-exposure. Eight equivalent samples were exposed to the deuterium flux from the capillary cracker $(2 \mathrm{sccm})$ for periods ranging from 1 to 8 hours. The resultant structural modification of the ML surfaces was measured by AFM using a Veeco Dimension 3100 scanning probe microscope in tapping mode with Si probes. The total D-content of these samples was determined by means of ERD using 3.5 MV Singletron accelerator (HVEE). The angle of incidence of the He beam was $15^{\circ}$ with respect to the sample surface. Recoiled hydrogen was detected by a Canberra passivated implanted planar silicon (PIPS) detector at an angle of $25^{\circ}$ with respect to the incident beam direction. A $9 \mu \mathrm{m}$ thick 
Mylar foil was mounted in front of the detector to block scattered He particles. Deuterium was utilized for enhanced sensitivity and, since the D-content in unexposed samples is negligible (in contrast to a native $\mathrm{H}$-content of $\sim 0.5$ at. $\%$ ), the determination of absolute uptake as a result of the exposure is more exact.

Resonant NRA measurements utilizing the ${ }^{1} \mathrm{H}\left({ }^{15} \mathrm{~N}, \alpha \gamma\right){ }^{12} \mathrm{C}$ reaction $[17,18]$ were performed on equivalent samples after exposure to a hydrogen flux $(1 \mathrm{sccm})$ for periods of 4 and 5 hours. The angle of incidence between the sample surface and the incoming beam was $15^{\circ}$. The amount of hydrogen trapped as a function of depth was derived from measuring the gamma radiation resulting from the resonance in the nuclear reaction at $6.385 \mathrm{MeV}$. Gamma rays were detected with a $4 \mathrm{x} 4$ inch bismuth germanate scintillation detector placed directly behind the sample. The correspondence between the incident NRA particle energy and the reaction depth was assigned on the basis of the layer profile derived from RBS measurements. These were performed with a $2 \mathrm{MeV}$ He beam at normal incidence to the sample surface. The spectra were registered at a scattering angle of $170^{\circ}$ with a Canberra PIPS detector. The ERD and RBS measurements shown in this report were acquired by AccTec BV, Eindhoven.

\section{Results}

We first consider the exposure time (i.e. fluence) dependence of the uptake and distribution of deuterium in the Mo/Si ML structure and its correlation with the consequent blister development. There was no obvious indication of physical damage to the surface of the sample exposed to the deuterium flux for 1 hour. All longer exposures resulted in a visible "discoloration" of the exposed region, which was a consequence of blister formation. AFM scans revealed the presence of a variety of blister sizes and shapes (single; double; multiple) on the surface. The structures observed were similar to those formed after comparable exposures

utilizing $\mathrm{H}_{2}$ [9]. The number and size of blisters increased with exposure time, and the extent of 
the "discoloration" was correlated with this increase. Figure 1(a)-(d) shows a series of "shaded image" representations of $10 \times 10 \mu \mathrm{m}^{2}$ AFM scans collected after exposure times of between 2 and 8 hours. This data is presented in this manner in order to highlight feature shapes and to enhance the visibility of smaller features. The z-ranges of the AFM scans used to generate these images were 70, 97, 126, and $125 \mathrm{~nm}$, respectively (see also Figure 2).

Two distinct blister size distributions are discernable on these images. It is evident that extending the exposure times leads to the development of increasingly complex double- and multi-blister structures. After the 2-hour exposure (figure 1(a)) most of the blisters in the larger size distribution already exhibit at least a double-blister structure. These become progressively more complex and distorted with exposure time leading to the very complex structures evident after the 8-hour exposure (figure 1(d)). 'Some blisters in the smaller size distribution also exhibit complex structures, but these are much less prevalent. Even after the 8-hour exposure a significant number of these blisters are still discrete, single-component features. An additional "third distribution" was also evident in the AFM scans. This distribution became clearly evident for exposure times $\geq 6$ hours and more than 1600 distinct features were identifiable on a $25 \times 25$ $\mu \mathrm{m}^{2}$ scan collected from the sample exposed for 8 hours. Its emergence is apparent by the small features (height $<15 \mathrm{~nm}$ ) that are evident in the "flat" regions of figure 1(d).

Figure 2 shows a plot of individual blister area versus height derived from analysis of of $25 \times 25 \mu \mathrm{m}^{2}$ AFM scans. Blisters are designated as belonging to either an "ion-induced" or a "thermal neutral" distribution. (The "third distribution" features were neglected for this analysis.) The designation used to identify the two distributions is derived from our previous report on hydrogen-induced blisters [9], in which two distinct distributions were also observed. In that case, comparison of the effects of different incident fluxes indicated that the smaller of the two was due to the action of energetic ions, while the larger was associated with hydrogenation via the incident thermal neutral flux. The designations "ion-induced" and 
"thermal neutral" are employed as short-hand labels-of-convenience. It should not be construed that the blisters in the two distributions develop entirely independent of each other. In fact, the presence of energetic ions has been observed to modify the development of the "thermal neutral" blisters. However, the validity of the generalized designations has been confirmed, and in particular that the formation of the smaller of the two does indeed require the presence of energetic ions. A manuscript aimed at elucidating the role of ions is current being finalized. Hence, their role will not be considered in detail in this report.

For exposure times $\leq 5$ hours the distinction between the two distributions was relatively clear-cut. The "ion-induced" blisters were smaller, had a different aspect ratio, and tended to have greater shape uniformity than the "thermal neutral" blisters. With extended exposure time, the dimensions of the blisters in both distributions exhibit increasing scatter and the distinction between the two becomes increasingly blurred. This is due to the emergence of the progressively more complex multi-blister features illustrated in Figure 1. Such structures can result in a significant change in area covered with little or no corresponding change of feature height. The reverse (i.e. a more dramatic change in feature height relative to area covered) can also occur in the form of "blister-on-blister" structures, several instances of which are visible on figure 1(d). These complex features introduce the increased scatter in the data points with increasing exposure time that is evident in figure 2 .

Figure 3 shows cross-sectional TEM images obtained from a D-exposed sample. Blistering is the result of delaminations associated with the two outermost Mo-on-Si interfaces. This is the same behavior as was observed in TEM images obtained from similar samples after H-exposure [9]. Double-blister structures in the "thermal neutral" distribution are the result of overlapping delaminations occurring at different Mo-on-Si interfaces, such as that shown in figure 3(b). The multi-blister structures present on the current samples may be more extreme manifestations of the same effect. 
The total D-content of the exposed samples was determined by ERD and the results are presented in figure 4(a). In all cases, the measured $\mathrm{D}$ was restricted to the outermost $1.7 \times 10^{17}$ at $/ \mathrm{cm}^{2}$ of the ML ( $\sim 30 \mathrm{~nm} ; \sim 4$ bilayers). The content increased in an approximately linear fashion during the first 3 hours of exposure. It peaked at $\sim 14.5 \times 10^{15}$ at. $/ \mathrm{cm}^{2}$ and subsequently stabilized at $\sim 12.8 \times 10^{15}$ at. $/ \mathrm{cm}^{2}$. This is comparable to the content that was determined after similar samples were exposed to a microwave-discharge deuterium plasma [19]. It was evident from the ERD measurements that the H-content of the samples also exhibited an initial increase and peak with increasing exposure time before stabilizing at a level that was $\sim 50 \%$ higher than that of an unexposed sample.

The remaining panels in figure 4 show the corresponding evolution of characteristic blister parameters. These are derived from distribution analyses such as those plotted in figure 2 . The "ion-induced" and the "thermal neutral" distributions are treated both separately and as a single combined distribution. Figure 4(b) shows the blister count data. No blisters were evident after the 1-hour exposure, but both distributions were present after all longer exposures. The total number of blisters remains constant for exposure times from 2 to 6 hours and then increases for longer exposures. The additional blisters that develop after 6 hours of exposure are attributed exclusively to the "ion-induced" distribution. Their increase coincides with the rapid emergence of the "third distribution" (which is not part of this blister count) suggesting that both features are related to a common underlying effect. The number of blisters designated as "thermal neutral" remains essentially unchanged for exposures of up to 8 hours.

Figure 4(c) shows that both distributions exhibit an initial increase in average blister height as a function of exposure time followed by a stabilization. The average height of the "ioninduced" blisters stabilized after 3 hours. In contrast, that of the "thermal neutral" blisters increased for the first 4 hours. Neither distribution showed any significant subsequent change in the average blister height. Figure 4(d) shows the percentage of the surface area covered by the 
blister distributions after each exposure. The combined data shows an initial growth period from 2-3 hours of exposure, a more gradual increase from 3-6 hours, and then accelerated growth for 7 and 8 hours. The area covered by "thermal neutral" blisters shows a continuous increase across the full exposure range studied. After an initial period of rapid growth during the first 3 hours of exposure, the area covered increases more gradually with extended exposure time. In contrast, the area covered by "ion-induced" blisters remains constant between the 3-hour and the 6-hour exposures, after which there is a second significant increase in the total area covered by blisters attributed to this distribution. This coincides with the renewed increase in blister number. However, there is no corresponding change in the average blister height. The reason for this is evident from figure 2(c) and (d). These panels indicate that for exposures $\geq 6$ hours additional blisters are "added" on both the lower and upper sides of the "ion-induced" distribution. The former signals the emergence of new blisters, while the latter indicates the resumption of growth (both laterally and in height) of existing blisters. The net effect is a relatively unchanged average blister height.

In order to obtain a picture of how hydrogen is distributed within the ML structure, samples were exposed using $\mathrm{H}_{2}$ as the feed gas and analyzed by resonant NRA. The results of measurements from an unexposed sample and from samples after 4- and 5-hour hydrogen exposures are shown in the figure 5. Due to the difference in flow rates, these exposures are roughly equivalent to 2 and 2.5 hours on the time scale of the $\mathrm{D}_{2}$ exposures. The approximate ML structure is indicated on the figure. The assignment of reaction depth to incident particle energy was based on the layer profile derived from RBS measurements, which have an accuracy of $\sim 5 \%$. Likely intermixed zones of $\sim 0.5 \mathrm{~nm}$ and $\sim 1 \mathrm{~nm}$ for the Si-on-Mo and Mo-on-Si interfaces, respectively, are also indicated.

An oscillatory variation in $\mathrm{H}$-content that correlates with the ML structure, with enhanced retention in Mo layers, is evident for all samples including as-deposited. After the 4- 
hour exposure, the H-content of all layers probed by NRA has increased substantially compared to the unexposed sample. The general shape of the profile has not been significantly altered, although the variation in the layer content has been enhanced. In contrast, a substantial change in the profile is evident after the 5-hour exposure, with the layers closer to the surface exhibiting enhanced uptake of $\mathrm{H}$. The oscillatory profile is still evident, but superimposed on a strong gradient as a function of depth. The content in the first Mo layers now peaks at $\sim 19$ at.\%. In addition, while the second (deeper) Mo layer now exhibits a lower $\mathrm{H}$-content than the Si layer immediately above it, there is an indication of a localized enhancement of the $\mathrm{H}$-content in the vicinity of the intervening interface.

H-contents of $\sim 7.5 \times 10^{15}$ at. $/ \mathrm{cm}^{2}$ and $\sim 1.2 \times 10^{16}$ at. $/ \mathrm{cm}^{2}$ were derived from the 4- and 5hour NRA data, respectively. These estimates are based on averaging the concentration of individual layers and subsequent summation of the derived contents. The corresponding Dcontents obtained from ERD of samples exposed for 2 and 3 hours (compensating for the difference in flow rate) are $\sim 1.0 \times 10^{16}$ at. $/ \mathrm{cm}^{2}$ and $\sim 1.4 \times 10^{16}$ at. $/ \mathrm{cm}^{2}$, respectively. The agreement between the two methods is reasonable. Interestingly, there is good agreement between the NRA- and ERD-derived contents for the longer exposure times, in spite of the shorter NRA depth range available for the 5-hour exposure. This indicates that the enhanced Huptake in the outermost layers that is evident in the NRA data between the 4- and 5-hour exposures is being replicated during the $\mathrm{D}$-uptake that occurs between the corresponding 2- and 3-hour exposures.

\section{Discussion}

\section{Native hydrogen in the MLs}

The NRA data from the as-deposited sample indicates a high $\mathrm{H}$-content at the surface with a strong gradient toward increasing depth in the outermost Si layer. The H-content 
measured in the buried layers also exhibits a decrease with increasing depth, particularly in the case of the Mo layers. Separate ERD analysis of an as-deposited sample yielded a H-content of $\sim 1.4 \times 10^{15}$ at. $/ \mathrm{cm}^{2}$, which was localized in the outermost $\sim 17 \mathrm{~nm}$ of the multilayer. This constitutes a local atomic concentration of $\sim 1.5 \%$. A surface hydrogen peak was also seen by Windt et al. in ERD measurements on Mo/Si MLs comparable to those used in the current study [20]. Since there was no indication of a significant deterioration in conditions during the final stages of deposition, we do not attribute the elevated near-surface content and the gradient in the H-profile of the outermost layers to the deposition process. Rather, these features are a consequence of exposure to ambient conditions. However, it cannot be ruled out that effects induced by the analysis beam contribute to the observed gradient. In this context it is noteworthy that the ERD analyses of the D-exposed samples tended to show a significant initial decrease in H-content (up to $\sim 57 \%$ ) upon onset of the irradiation. This was attributed to the removal of adsorbed molecules (e.g. water) due to beam-induced heating of the samples (up to $\left.\sim 70{ }^{\circ} \mathrm{C}\right)$. However, the effect was not observed from the 1-hour D-exposed sample and it became more pronounced with increased exposure times. It was also not observed during ERD analysis of unexposed and $\mathrm{H}$-exposed samples. This suggests that the D-exposures induced structural and/or chemical composition modifications to the surface that increased the carrying capacity for adsorbed species.

The H-contents determined by NRA in Si and Mo at depths $>15 \mathrm{~nm}(\sim 0.2$ at. $\%$ and $\sim 0.6$ at. $\%$, respectively) are better representations of the H-content that arises during the sample deposition. The primary source of this hydrogen is water in the vacuum system, which is the dominant component in the residual gas before deposition commences. The O- and C-contents of the samples currently under investigation, as determined by X-ray photo-electron spectroscopic depth profiling, were below the limits of detection (1 and 2 at.\%, respectively). Windt et al. [20] studied the effect of initial background pressure (also predominantly $\mathrm{H}_{2} \mathrm{O}$ ) in 
the range $1.33 \times 10^{-3}$ to $8 \times 10^{-6} \mathrm{~Pa}$. The $\mathrm{O}$ content of their MLs was found to be $\sim 0.5$ at. $\%$ while the $\mathrm{C}$ content was below the limit of detection $(\sim 0.1$ at. $\%)$, with neither content being influenced by the initial background pressure. In contrast, they found a linear scaling of the $\mathrm{H}$ content of their samples with background pressure. Their reported average H-content of $\sim 0.25$ at. $\%$ for the ML deposited at the lowest starting pressure $\left(\sim 8 \times 10^{-6} \mathrm{~Pa}\right)$ is comparable to that indicated by the NRA data for the deeper layers of our samples. The better starting base pressure in our case is partially offset by an approximately 5 times longer deposition time as compared to that of Windt et al.

H-uptake during deposition of thin films can have a significant influence on their properties [21-23]. In the specific case of $\mathrm{Mo} / \mathrm{Si} \mathrm{MLs}$, increasing $\mathrm{H}$-content resulted in more compressive Si layers and more tensile Mo layers, with the effect on the Mo layers being $~ 2.5$ greater than that on the Si layers. [20]. All samples used in this study were prepared simultaneously ensuring that there were no variations in deposition conditions between samples. Furthermore, there is no indication that the presence of native hydrogen in the MLs plays a significant role in the development of blisters. Attempts to induce blistering by heating samples in vacuum and in $\mathrm{a}_{2}$ atmosphere produced no effect. Active irradiation by $\mathrm{H}$ atoms and/or hydrogen ions was necessary in order to produce blisters.

\section{Hydrogen uptake and distribution}

Broadly speaking, the result of increasing the $\mathrm{D}$ exposure time is an initial increase followed by stabilization of the content in the sample. However, the content appears to pass through a small maximum around 3-to-4 hours of exposure before stabilizing at $\sim 1.3 \times 10^{16}$ at. $/ \mathrm{cm}^{2}$ (see Fig. 4(a)). The same trend is observed in the H-content of these samples, with the $\mathrm{H}$ presumably being sourced from hydrogen-containing molecules in the apparatus and on the surface. The simultaneous increase and coincident peaks of the $\mathrm{H}$ - and $\mathrm{D}$-contents suggest that 
synergistic uptake and layer modification effects that alter the maximum "hydrogen" content that can be retained by the ML structure are operative. The effect is transient, with the accumulated $\mathrm{H}$ - and D-contents decreasing somewhat with extended time. None-the-less, the amount of $\mathrm{H}$ in all $\mathrm{D}$-exposed samples remains elevated relative to that of an as-deposited sample.

The flux incident on the surface was dominated by thermal atoms and molecules, while containing a minor fraction $(<0.0001 \%)$ of energetic $(>800 \mathrm{eV})$ ions [9]. Hydrogenation of a sample via the uptake and diffusion of thermal species should tend to produce a decreasing gradient from the surface. In contrast, the ions are capable of penetrating through several layers of the ML structure. Hence the expectation, at least during the early stages of exposure, is that hydrogenation via thermal neutrals should favour uptake by the outermost layers, while ion implantation should play a more important role in the deeper, buried layers.

The relatively flat NRA profile after the 4-hour exposure indicates that the H-content arising from direct implantation and associated ion-induced effects (e.g. facilitated transport of thermal species to deeper layers) is competitive with that arising from thermal processes during the initial period. Although the fraction of ions is extremely small, their high energy ensures a much higher uptake probability relative to thermal species. The oscillatory variation observed after 4 hours is not inconsistent with simple ion implantation. SRIM calculations using $850 \mathrm{eV}$ $\mathrm{H}^{+}$ions also exhibit preferential $\mathrm{H}$ accumulation in Mo layers, which is due to the relative material stopping powers.

In contrast, the profile change that occurs between the 4- and 5-hour hydrogen exposures is inconsistent with an ion-dominated process. The strong gradient in H-content as a function of sample depth indicates that processes involving thermal neutrals become progressively more important with extended exposure time and dominate the latter stages of $\mathrm{H}$ uptake prior to stabilization of the content. These processes strongly favour uptake by the 
outermost Mo layer. Furthermore, the depth profile suggests that the Mo-on-Si interface may act as a significant hindrance to transport to deeper layers. Blocking of hydrogen penetration to deeper layers by the Mo-on-Si interface may be related to the strain in the silicide interface that forms between the two materials (see 'Blister development'). Yamashita et al. reported that a strained SiGe cap layer could be utilized to prevent penetration during plasma hydrogenation of Si [24]. A compressively-strained buried SiGe layer can be used to create a localized hydrogen content reduction [14], while hydrogen accumulates in one that is tensilely-strained [15]. The lack of Hcontent increase in deeper layers between 4 and 5 hours further suggests that ion-driven uptake of hydrogen ceases to be effective once a certain fluence limit has been reached and/or that the maximum $\mathrm{H}$-content that can be achieved via ion-driven processes is significantly lower than that which can be realized via thermal hydrogenation processes.

H-retention by the Si layers is almost certainly dominated by passivation of Si dangling bonds. a-Si layers deposited in a hydrogen atmosphere [25] or subjected to post-deposition hydrogen ion treatment [26] can accommodate H-contents of up to 30 at.\%. In both cases, contents of up to $\sim 20$ at. $\%$ could be achieved with only a minor decrease in the layer density relative to non-hydrogenated $a$-Si. Thus, the content levels reached in the current case would not necessitate a significant expansion of the as-deposited layers. The activation energy for $\mathrm{H}$ diffusion in $a$-Si is reported as $\sim 1.5 \mathrm{eV}$, as compared with $\mathrm{Si}-\mathrm{H}$ bond strengths of up to $3.5 \mathrm{eV}$ $[27,28]$. Consequently, passivated dangling bonds should represent a stable sink for hydrogen in the Si layers. Based on ab initio MD simulations, Su and Pantelides identified a floating bond-H complex as the "migrating species" for H-diffusion in a-Si [28], while Abtew et al. identified a somewhat similar "fluctuating bond center detachment" mechanism for release of hydrogen bonded within a Si network [29]. Both mechanisms require an active site in the form of an under-coordinated Si atom for the diffusion of $\mathrm{H}$ through the layer. The availability of such sites in the as-deposited layer is determined by the deposition conditions. Part of the influence of 
ions contributing to the emergence of the different blister distributions seen in the current samples may be due to enhancement of diffusion through the Si layers via defect introduction and cleavage of existing bonds, thereby creating new active sites.

The $\mathrm{H}$ concentration observed in the outermost Mo layer is remarkably high. By way of comparison, retention on the order of several at. $\%$ has been reported for Mo and W samples exposed to hydrogen isotopes $[30,31]$. The current result is also higher than the $\mathrm{H}$ retention by disordered nano-crystalline $\mathrm{W}$ films ( 9 at.\%) [32] and is comparable to the maximum $\mathrm{H}$ concentration observed after co-deposition of $\mathrm{W}$ and $\mathrm{H}(\sim 16$ at. $\%)$ [33]. The retention of $\mathrm{H}$ by Mo is particularly surprising in light of its positive heat of solution [34]. This is highlighted by the distribution of hydrogen in $\mathrm{Mo} / \mathrm{V}$ single crystal superlattices $[35,36]$ in which localization is exclusively in the V layers (V having a negative heat of solution). Somewhat closer to the current MLs are $\mathrm{Nb} / \mathrm{Ta}$ lyered superlattices $[37,38]$. These are characterized as "weakly modulated" since both materials have an affinity for hydrogen. The Mo/Si system can be considered as an inverse case since neither bulk material is a natural adsorber of hydrogen. A notable difference between metal/metal and metal/semiconductor systems is the potential for a strong chemical interaction between the materials in the latter case.

The polycrystalline nature of the Mo layers in the current samples will contribute to their ability to retain of hydrogen. A comparative study on the retention of D by single crystal and polycrystalline Mo indicated a 2 orders of magnitude greater uptake by the bulk in the latter case [30], although the absolute amount remained low (on the order of $10^{-2}$ at.\%). The chemical state of Mo did not appear to have an influence on the ability to incorporate $\mathrm{H}$, with a $\mathrm{MoO}_{3}$ layer exhibiting a similar retention to that of polycrystalline Mo. Rigato et al. indicated that hydrogen was not retained by thick Mo layers deposited in $\mathrm{Ar} / \mathrm{H}_{2}$ atmospheres containing up to $10 \% \mathrm{H}_{2}$ [25]. However, in thin film MLs the stress in the individual layers and the stress differences between layers can play a role in determining hydrogen uptake. Mo layers in Mo/Si MLs with 
layer thicknesses in the 3-5 nm range typically exert tensile stress [20, 39-42]. In contrast, thick Mo layers exhibit compressive stress [41]. Mo layers deposited on $a$-Si layers exhibit an amorphous-to-crystalline transition at a thickness of $\sim 2 \mathrm{~nm}$ and a scaling of the crystallite size with layer thickness above that point [43]. The polycrystalline structure of the Mo layers [44] with the small size of Mo nano-crystallites $(\sim 3 \mathrm{~nm})$ [45], allows for enhanced trapping in vacancies and at grain boundaries and crystallite faces. This represents an example of a material constrained on the nano-scale exhibiting properties substantially different from those of the corresponding "bulk" [46]. The high content in the outermost Mo layer is likely due to trapping in vacancies and voids in the structure. First principle calculations indicate that a single Mo mono-vacancy can accommodate up to $12 \mathrm{H}$ atoms, with the added possibility of $\mathrm{H}_{2}$ formation $[47,48]$. Hydrogen accumulated in this way can more readily provide a reservoir to feed blister growth than hydrogen that is chemically bound in the Si layers.

\section{Blister development}

Figure 4(b) illustrates that the initial number density of both "ion-induced" and "thermal neutral" blisters is established well before the D-content reaches a stable value. In neither case is the number of blisters formed a simple function of the D-content. In the case of the "thermal neutral" blisters, pre-existing nucleation sites introduced during sample deposition are likely to play a crucial role in nucleation. Since the number of such sites will be determined by the sample preparation methods, the number of associated blisters that can be formed is inherently limited. In contrast, the creation of new nucleation sites by incident energetic ions should be related to the total ion fluence received. None-the-less, figure 4(b) demonstrates that a fluence limit also exists in this case. The early establishment of the number density of "ion-induced" blisters suggests that the processes involved are subject to competition between blister activation/nucleation, on the one hand, and active blister growth, on the other. Growth of 
existing blisters may dominate over the emergence of new blisters, for instance via Ostwald ripening, once a sufficient density of active sites has been realised.

Considering the data shown in figure 4(c), the average height of "ion-induced" blisters stabilizes after 3 hours, correlating directly with the stabilization of the D-content. In contrast, the average height of the "thermal neutral" blisters continues to increase during the first 4 hours of exposure. This suggests that growth of the latter has a greater dependence on (re-)distribution of D-species within the ML structure occurring on a longer timescale than the D-uptake. While the average height of the "thermal neutral" blisters remains stable for the 4-hour and longer exposures, the percentage surface area covered by such blisters continues to increase with each extended exposure time (figure 4(d)). This on-going growth also suggests that internal redistribution (e.g. transport; segregation to interfaces) factors significantly in the development of these blisters.

The additional lateral growth reflects the emergence of the increasingly complex blister structures seen in figure 1. As noted in the Results section, the "thermal neutral" blisters are more prone to develop these structures than the "ion-induced" blisters. This difference points to another distinction between the two distributions. The relatively infrequent development of complex structures among the "ion-induced" distribution suggests that their formation is a random process arising from the incidental overlap of otherwise independent blisters. In contrast the early and widespread development of double-blisters among the "thermal neutral" distribution points to an active and dependent connection between the blister components. In light of the TEM image illustrating that these blister components originate at different interfaces (figure 3(b)), this implies highly-localized transport of hydrogen species between the components (i.e. across the bilayer structure).

The blistering phenomena observed in the current case have obvious similarities with techniques utilized in relation to silicon-on-insulator transfer technology. The original process 
employs a defined-energy ion beam to cleave a Si sample at a desired depth [10]. However, the use of buried strain layers for localizing detachment $[12,13]$ is more relevant to the current study. In these cases a buried, compressively-strained SiGe layer acts initially as a strong sink for vacancies leading to subsequent accumulation of platelets oriented along the layer plane. Consequently, layer delamination is initiated while avoiding straggling effects typically associated with energetic ions. Blister nucleation in ion-implanted Si wafers is driven by the formation of passivated (001) platelets [49-51], with in-plane compressive stress and out-of-plane tensile strain inducing the layer separation [51-53]. In the case of a Si/SiGe/Si heterostructure for layer transfer purposes, peaks in the out-of-plain strain developed at both interfaces [12].

In the current samples the interfaces between the Mo and Si layers appear to act as preferred nucleation points for the accumulation of vacancies and mobile hydrogen species. Detachment and the development of macroscopic blisters occur preferentially in the vicinity of the Mo-on-Si interfaces, while detachment at Si-on-Mo interfaces was not observed. None-theless, the spike at $\sim 12 \mathrm{~nm}$ depth in the NRA data for the 5-hour exposure indicates accumulation of hydrogen in the vicinity of this interface. A similar enhancement is not evident at the outermost Si-on-Mo interface, although it may be masked by the high H-content in the "bulk" of the Mo layer. Macroscopically, the as-deposited Mo and Si layers in ML structures such as those currently under investigation are typically tensile and compressive, respectively [20, 39-42, 54]. While the Si layers are amorphous, the Mo layers exhibit an amorphous-to-polycrystalline transition at a thickness of $\sim 2 \mathrm{~nm}[43,45]$. Zubarev et al. identified a $(1-2 \mathrm{~nm})$ cluster form of Mo, which developed at nominal layer thicknesses between 1.5 and $1.9 \mathrm{~nm}$ after formation of a silicide layer, as a transient state between the amorphous and polycrystalline ones [55]. The MoSi interfaces are symmetric for Mo layer thicknesses $<2 \mathrm{~nm}$, whereas asymmetric zones develop in the case of thicker layers due to reduced silicide formation when $\mathrm{Si}$ is deposited on polycrystalline Mo $[43,56,57]$. Above the transition thickness, the crystallites do not exhibit a 
preferred growth orientation and their size is closely correlated with the Mo layer thickness [45, 58]. The latter point suggests that the effect of crystallization is "retroactive" on the already deposited Mo and thus that the crystallites can extend down to the underlying interfacial layer.

Stress in deposited MLs is linked to internal strain in Mo crystallites due to lattice mismatch originating during growth at the interfaces [58]. In the current case the localization of mobile vacancies and hydrogen species and the subsequent manifestation (or not) of macroscopic blister will be determined by the stress states introduced during silicide formation at the two interfaces, with the alternating interfaces developing from a starting point of $a$-Si and polycrystalline Mo. In the former case an initially stabilized amorphous silicide layer transitions to polycrystalline Mo, while in the latter it returns to $a$-Si.

The development of stress in Mo layers grown on $a$-Si is strongly dependent on the layer thickness $[55,59]$. Factors influencing the development of stressed state in the Mo layer were identified as bombardment by energetic particles, generating biaxial compressive stress, and recrystallization, which introduces tensile stress [59]. With reference to the published work in relation to the effect of buried strained SiGe layers on hydrogen localization $[14,15]$, blister formation localized near the Mo-on-Si interface coupled with $\mathrm{H}$ accumulation at the Si-on-Mo interface points to determining influences of compressive and tensile strained centres within the respective interfacial layers. The former would tend to accumulate vacancies while blocking thermal transport of $\mathrm{H}$ to deeper layers, while the latter would getter hydrogen.

The main effect of the ion polishing step applied to the as-deposited Si layers is the reduction of interface roughness [16]. Since the Si layers are amorphous, ion treatment will not dramatically alter the material structure. Sufficiently high energy ion irradiation can modify the buried Si-on-Mo interface resulting in an increased interfacial thickness and chemical composition [16]. The energy employed during the current depositions is insufficient to induce 
such effects. However, noble gas atoms implanted near the top of the Si layer may act as blister nucleation points and thus may contribute to the localization of blistering that we observed.

\section{Effects of extended exposure}

The trends in the "ion-induced" distributions shown in figure 4(b) and (d) indicate that the $\mathrm{ML}$ is prone to successive distinct stages of blistering as a result of exposure to energetic ions. The first stage ends with the initial stabilization of the D-content and the "ion-induced" blisters remain constant in terms of number density, average height, and area covered for a period after this point has been reached. Resumption of blister formation attributed to "ioninduced" processes occurs between the 6- and 7-hour exposures. This may indicate a second fluence threshold arising from blister initiation at deeper levels in the sample. Based on current and previous [9] TEM cross-sections, the detachment points formed during the first 4 hours of exposure are localized at the two outermost Mo-on-Si interfaces. Slower accumulation of deuterium in deeper layers, coupled with preferential consumption by active sites in the overlaying bilayers may delay the manifestation of blistering at deeper Mo-on-Si interfaces. The coincident increase in the number of multi-blister structures in the "ion-induced" distribution is consistent with the emergence of new overlapping delamination points.

The rapid development of the "third distribution" blisters coincides with renewed development and growth of "ion-induced" blisters. Since the latter suggests the existence of a second ion fluence threshold, the development of the "third distribution" may be an associated ion-induced effect. One possibility is that it represents the emergence of delayed (relative to the Mo-on-Si interfaces) blister formation at the Si-on-Mo interfaces or within the individual layers. The renewed blistering does not appear to coincide with an appreciable increase in the measured D-content. At best, a slight increase is evident after the 8-hour exposure. This suggests that the resumption of blister development is mainly the result of redistribution within the ML structure 
and that only a small fraction of the total D-content may be sufficient to induce the additional blistering stage. The fraction of incorporated atoms that ultimately contribute to blistering of silicon is typically relatively small [51]. Thus, implantation/transport of a small amount of deuterium to deeper layers could allow blister development without a substantial increase in the total content.

\section{Conclusion}

We have studied the correlation between $\mathrm{D}$-uptake by $\mathrm{Mo} / \mathrm{Si} \mathrm{ML}$ samples and the growth and number density of blisters formed as a consequence. Hydrogen-induced blistering of these samples is complex and multi-faceted. Various blister development processes are simultaneously operative across different depth and time scales. Two distinct distributions, attributed to the effects of energetic ion irradiation and thermal D uptake, are tracked as a function of exposure time. The effects observed cannot be accounted for simply on the basis of the total hydrogen content in the sample. The number of "ion-induced" blisters is established well before stabilization of the D-content, although their size is directly correlated with it. The number of "thermal neutral" blisters is also established before content stabilization. However, these continue to grow in lateral size, via the development of blister structures of increasing complexity, even after the content has stabilized. In this case, relatively slow internal redistribution processes must play a more significant role in blister growth and localized transport across the ML structure is inferred.

NRA depth profiling of $\mathrm{H}$-exposed samples reveals preferential accumulation in the Mo layers. The profiles indicate that processes induced by the minority ion fraction are competitive with direct uptake of thermal neutrals during the early stages of exposure, while thermal processes come increasingly to the fore with extended exposure time. The latter produce a strong enhancement of the H-content in the outermost Mo layer, reaching a maximum of $\sim 19$ 
at.\%. This content is made possible by the polycrystalline nature of the Mo layer with small crystallite sizes, which allows for numerous defects, voids, and vacancies that can accommodate multiple $\mathrm{H}$ atoms. Preferential detachment at the Mo-on-Si interfaces together with the indication of $\mathrm{H}$-accumulation at the Si-on-Mo interface is attributed to the introduction of compressive and tensile strained centres in the interfacial layers during deposition.

Extended exposures indicate that the ML structure is susceptible to successive stages of blistering via ion-induced mechanisms. These may be due to development of blisters at deeper interfaces in the samples, at the Si-on-Mo interfaces, or within the individual layers. A major challenge remains to study how the underlying processes operate in isolation and the extent of synergistic effects when the incident flux contains a mixture of neutral and ionic species.

\section{Acknowledgements}

TEM sample preparation and imaging was performed by Dr. Enrico G. Keim of the $\mathrm{MESA}^{+}$Institute, University of Twente. The NRA measurements were performed at the Institute of Ion Beam Physics and Materials Research, HZDR (Helmholtz-Zentrum DresdenRossendorf) in Dresden by Dr. René Heller. Dr. Wim Arnold Bik is gratefully acknowledged for his advice on and assistance with the RBS, ERD, and NRA measurements. DIFFER is part of the 'Stichting voor Fundamenteel Onderzoek der Materie (FOM)', which is financially supported by the 'Nederlandse Organisatie voor Wetenschappelijk Onderzoek (NWO)'. This work was part of the FOM Industrial Partnership Programme I10 "Extreme UV multilayer Optics" ("XMO") with additional funding from Agentschap NL through the "EXEPT" programme. 


\section{References}

[1] P.T. Hammond, Adv. Mater. 16/15 (2004) 1271.

[2] E. Louis, A.E. Yakshin, T. Tsarfati, F. Bijkerk, Prog. Surf. Sci. 86 (2011) 255.

[3] B. Hjörvarsson, G. Andersson, E. Karlsson, J. Alloy. Compd. 253-254/0 (1997) 51.

[4] F. Klose, C. Rehm, D. Nagengast, H. Maletta, A. Weidinger, Phys. Rev. Lett. 78/6 (1997) 1150 .

[5] K. Munbodh, F.A. Perez, C. Keenan, D. Lederman, M. Zhernenkov, M.R. Fitzsimmons, Phys. Rev. B 83/9 (2011) 094432.

[6] K. Munbodh, F.A. Perez, D. Lederman, J. Appl. Phys. 111 (2012) 123919.

[7] C. Frigeri, M. Serenyi, N.Q. Khanh, A. Csik, F. Riesz, Z. Erdelyi, L. Nasi, D.L. Beke, H.G. Boyen, Nanoscale Research Letters 6 (2011) 186.

[8] M.G. Pelizzo, A.J. Corso, P. Zuppella, D.L. Windt, G. Mattei, P. Nicolosi, Optics Express 19/16 (2011) 14838.

[9] A.S. Kuznetsov, M.A. Gleeson, F. Bijkerk, J. Phys.: Condens. Matter 24/5 (2012) 052203.

[10] M. Bruel, Nucl. Instrum. Methods Phys. Res. Sect. B-Beam Interact. Mater. Atoms 108/3 (1996) 313.

[11] G.K. Celler, S. Cristoloveanu, J. Appl. Phys. 93/9 (2003) 4955.

[12] L. Shao, Y. Lin, J.K. Lee, Q.X. Jia, Y. Wang, M. Nastasi, P.E. Thompson, N.D.

Theodore, P.K. Chu, T.L. Alford, J.W. Mayer, P. Chen, S.S. Lau, Appl. Phys. Lett. 87/9 (2005) 091902.

[13] F. Okba, N. Cherkashin, Z. Di, M. Nastasi, F. Rossi, A. Merabet, A. Claverie, Appl. Phys. Lett. 97 (2010) 031917.

[14] F.J. Henley, M.A. Brayan, W.G. En, US Patent No. 6,500,732, Dec 312002.

[15] A.J. Pitera, E.A. Fitzgerald, J. Appl. Phys. 97/10 (2005) 104511.

[16] I. Nedelcu, R. de Kruijs, A.E. Yakshin, F. Tichelaar, E. Zoethout, E. Louis, H. Enkisch, S. Muellender, F. Bijkerk, Thin Solid Films 515/2 (2006) 434.

[17] W.A. Lanford, H.P. Trautvetter, J.F. Ziegler, J. Keller, Appl. Phys. Lett. 28/9 (1976) 566.

[18] P. Trocellier, C. Engelmann, J. Radioanal. Nucl. Chem. 100 (1986) 117.

[19] A.S. Kuznetsov, R.W.E. van de Kruijs, M.A. Gleeson, K. Schmid, F. Bijkerk, J. Surf. Investig.- X-ray Syncro. 4/4 (2010) 563.

[20] D.L. Windt, W.L. Brown, C.A. Volkert, W.K. Waskiewicz, J. Appl. Phys. 78/4 (1995) 2423.

[21] J.M. Schneider, A. Anders, B. Hjörvarsson, I. Petrov, K. Macak, U. Helmersson, J.E. Sundgren, Appl. Phys. Lett. 74/2 (1999) 200.

[22] J.M. Schneider, B. Hjörvarsson, X. Wang, L. Hultman, Appl. Phys. Lett. 75/22 (1999) 3476 .

[23] J.M. Schneider, K. Larsson, J. Lu, E. Olsson, B. Hjörvarsson, Appl. Phys. Lett. 80/7 (2002) 1144.

[24] Y. Yamashita, Y. Sakamoto, Y. Kamiura, T. Ishiyama, Jpn. J. Appl. Phys. Part 1 - Regul. Pap. Brief Commun. Rev. Pap. 46/4A (2007) 1622.

[25] V. Rigato, A. Patelli, G. Maggioni, G. Salmaso, V. Mattarello, M.G. Pelizzo, P. Nicolosi, L. Depero, E. Bontempi, P. Mazzoldi, Surf. Coat. Technol. 174 (2003) 40.

[26] R. Schlatmann, A. Keppel, Y. Xue, J. Verhoeven, C.H.M. Maree, F. Habraken, J. Appl. Phys. 80/4 (1996) 2121.

[27] P.A. Fedders, Phys. Rev. B 61/23 (2000) 15797.

[28] Y.S. Su, S.T. Pantelides, Phys. Rev. Lett. 88/16 (2002) 165503.

[29] T.A. Abtew, F. Inam, D.A. Drabold, Epl 79/3 (2007) 36001.

[30] V.K. Alimov, J. Roth, S. Lindig, J. Nucl. Mater. 381/3 (2008) 267. 
[31] M.H.J. 't Hoen, B. Tyburska-Püschel, K. Ertl, M. Mayer, J. Rapp, A.W. Kleyn, P.A. Zeijlmans van Emmichoven, Nucl. Fusion 52/2 (2012) 023008.

[32] V. Nemanic, B. Zajec, D. Dellasega, M. Passoni, J. Nucl. Mater. 429 (2012) 92.

[33] G. De Temmerman, R.P. Doerner, J. Nucl. Mater. 389/3 (2009) 479.

[34] R. Griessen, Phys. Rev. B 38/6 (1988) 3690.

[35] B. Hjörvarsson, J. Ryden, E. Karlsson, J. Birch, J.E. Sundgren, Phys. Rev. B 43/8 (1991) 6440 .

[36] B. Hjörvarsson, M. Vergnat, J. Birch, J.E. Sundgren, B. Rodmacq, Phys. Rev. B 50/15 (1994) 11223.

[37] P.F. Miceli, H. Zabel, J.E. Cunningham, Phys. Rev. Lett. 54/9 (1985) 917.

[38] M. Wolff, G.K. Palsson, P.T. Korelis, J.A. Dura, C. Majkrzak, B. Hjorvarsson, J. Phys.:Condes. Matter 24/25 (2012) 255306.

[39] J.M. Freitag, B.M. Clemens, Appl. Phys. Lett. 73/1 (1998) 43.

[40] P.B. Mirkarimi, Optical Engineering 38/7 (1999) 1246.

[41] D.L. Windt, J. Vac. Sci. Technol. A-Vac. Surf. Films 18/3 (2000) 980.

[42] E. Zoethout, G. Sipos, R. van de Kruijs, A. Yakshin, E. Louis, S. Muellender, F. Bijkerk, Proc. SPIE 5037 (2003) 872.

[43] S. Bajt, D.G. Stearns, P.A. Kearney, J. Appl. Phys. 90/2 (2001) 1017.

[44] V.I.T.A. De Rooij-Lohmann, A.E. Yakshin, R.W.E. Van De Kruijs, E. Zoethout, A.W. Kleyn, E.G. Keim, M. Gorgoi, F. Schafers, H.H. Brongersma, F. Bijkerk, J. Appl. Phys. 108/1 (2010) 014314.

[45] I. Nedelcu, R.W.E. van de Kruijs, A.E. Yakshin, F. Bijkerk, Phys. Rev. B 76/24 (2007) 245404.

[46] H. Gleiter, Mater. Trans. 44/6 (2003) 1057.

[47] K. Ohsawa, K. Eguchi, H. Watanabe, M. Yamaguchi, M. Yagi, Phys. Rev. B 85/9 (2012) 094102.

[48] Y.W. You, X.S. Kong, X.B. Wu, Y.C. Xu, Q.F. Fang, J.L. Chen, G.N. Luo, C.S. Liu, B.C. Pan, Z.G. Wang, AIP Adv. 3/1 (2013) 012118.

[49] J. Grisolia, G. Ben Assayag, A. Claverie, B. Aspar, C. Lagahe, L. Laanab, Appl. Phys. Lett. $76 / 7$ (2000) 852.

[50] S. Romani, J.H. Evans, Nucl. Instrum. Methods Phys. Res. Sect. B-Beam Interact. Mater. Atoms 44/3 (1990) 313.

[51] B. Terreault, Phys. Status Solidi A 204/7 (2007) 2129.

[52] M. Nastasi, T. Hochbauer, J.-K. Lee, A. Misra, J.P. Hirth, M. Ridgway, T. Lafford, Appl. Phys. Lett. 86/15 (2005) 154102.

[53] J.K. Lee, Y. Lin, Q.X. Jia, T. Hochbauer, H.S. Jung, L. Shao, A. Misra, M. Nastasi, Appl. Phys. Lett. 89/10 (2006-3) 101901.

[54] R.R. Kola, D.L. Windt, W.K. Waskiewicz, B.E. Weir, R. Hull, G.K. Celler, C.A. Volkert, Appl. Phys. Lett. 60/25 (1992) 3120.

[55] E.N. Zubarev, V.V. Kondratenko, Y.P. Pershyn, V.A. Sevryukova, Thin Solid Films 520/1 (2011) 314.

[56] S. Yulin, T. Feigl, T. Kuhlmann, N. Kaiser, A.I. Fedorenko, V.V. Kondratenko, O.V. Poltseva, V.A. Sevryukova, A.Y. Zolotaryov, E.N. Zubarev, J. Appl. Phys. 92/3 (2002) 1216.

[57] S. Bruijn, R.W.E. van de Kruijs, A.E. Yakshin, F. Bijkerk, Deff. Diff. Forum 283-286 (2009) 657.

[58] R.W.E. van de Kruijs, E. Zoethout, A.E. Yakshin, I. Nedelcu, E. Louis, H. Enkisch, G. Sipos, S. Mullender, F. Bijkerk, Thin Solid Films 515/2 (2006) 430.

[59] V.I. Pinegyn, E.N. Zubarev, V.V. Kondratenko, V.A. Sevryukova, S.A. Yulin, T. Feigl, N. Kaiser, Thin Solid Films 516/10 (2008) 2973. 

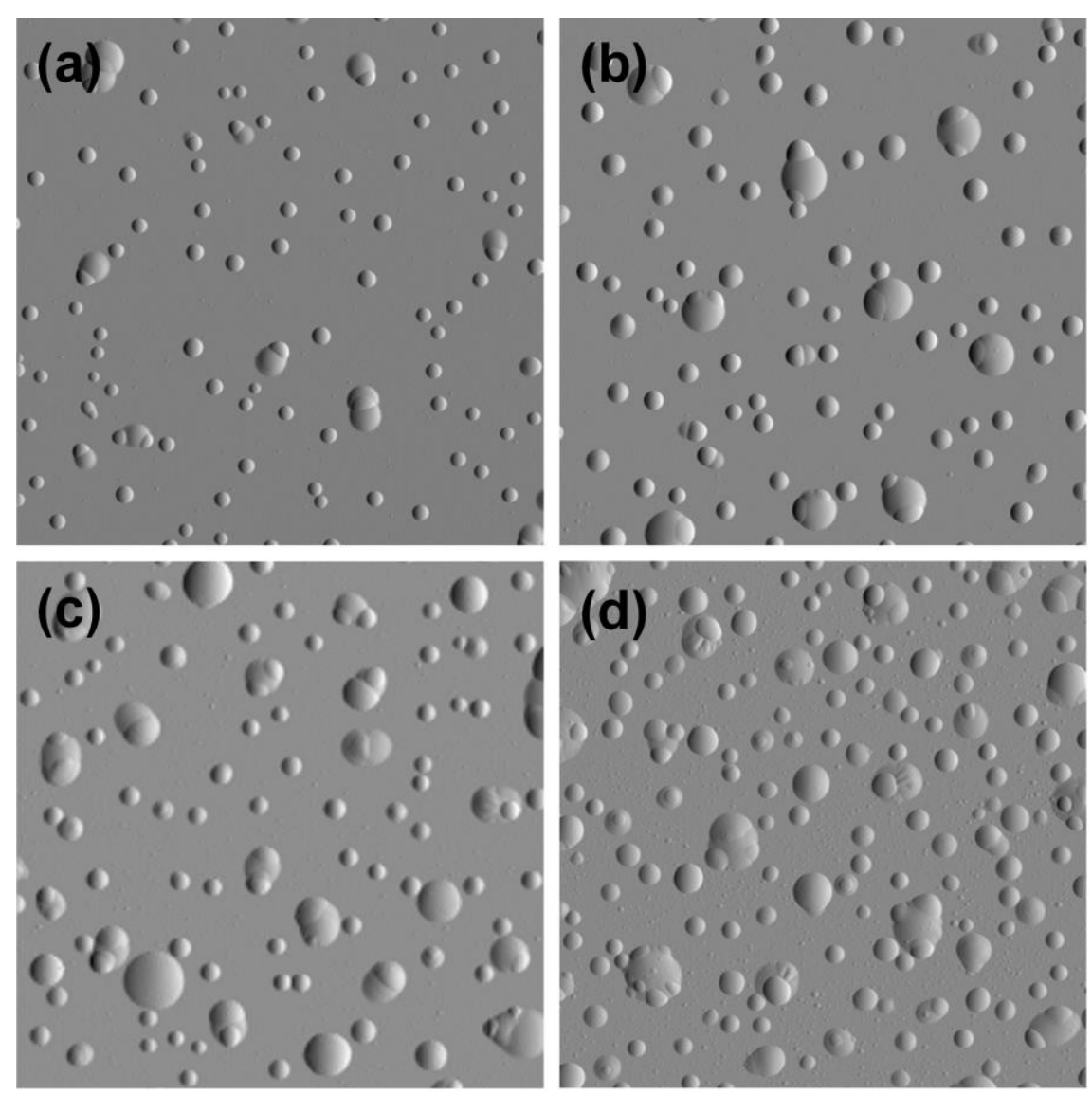

Figure 1: Shaded-image representations of $10 \times 10 \mu \mathrm{m}^{2}$ AFM scans collected from samples after exposure to the deuterium flux for (a) 2 hours, (b) 4 hours, (c) 6 hours, and (d) 8 hours. 
A.S. Kuznetsov, M.A. Gleeson, F. Bijkerk,

Thin Solid Films, 545, 571-579, (2013),

http://dx.doi.org/10.1016/j.tsf.2013.07.039


Figure 2: Plots of individual blister area versus the corresponding blister height derived from $25 \times 25 \mu \mathrm{m}^{2}$ AFM images. The blisters are assigned as either "ioninduced" (filled circles) or "thermal neutral" (open circles). 
A.S. Kuznetsov, M.A. Gleeson, F. Bijkerk,

Thin Solid Films, 545, 571-579, (2013),

http://dx.doi.org/10.1016/j.tsf.2013.07.039
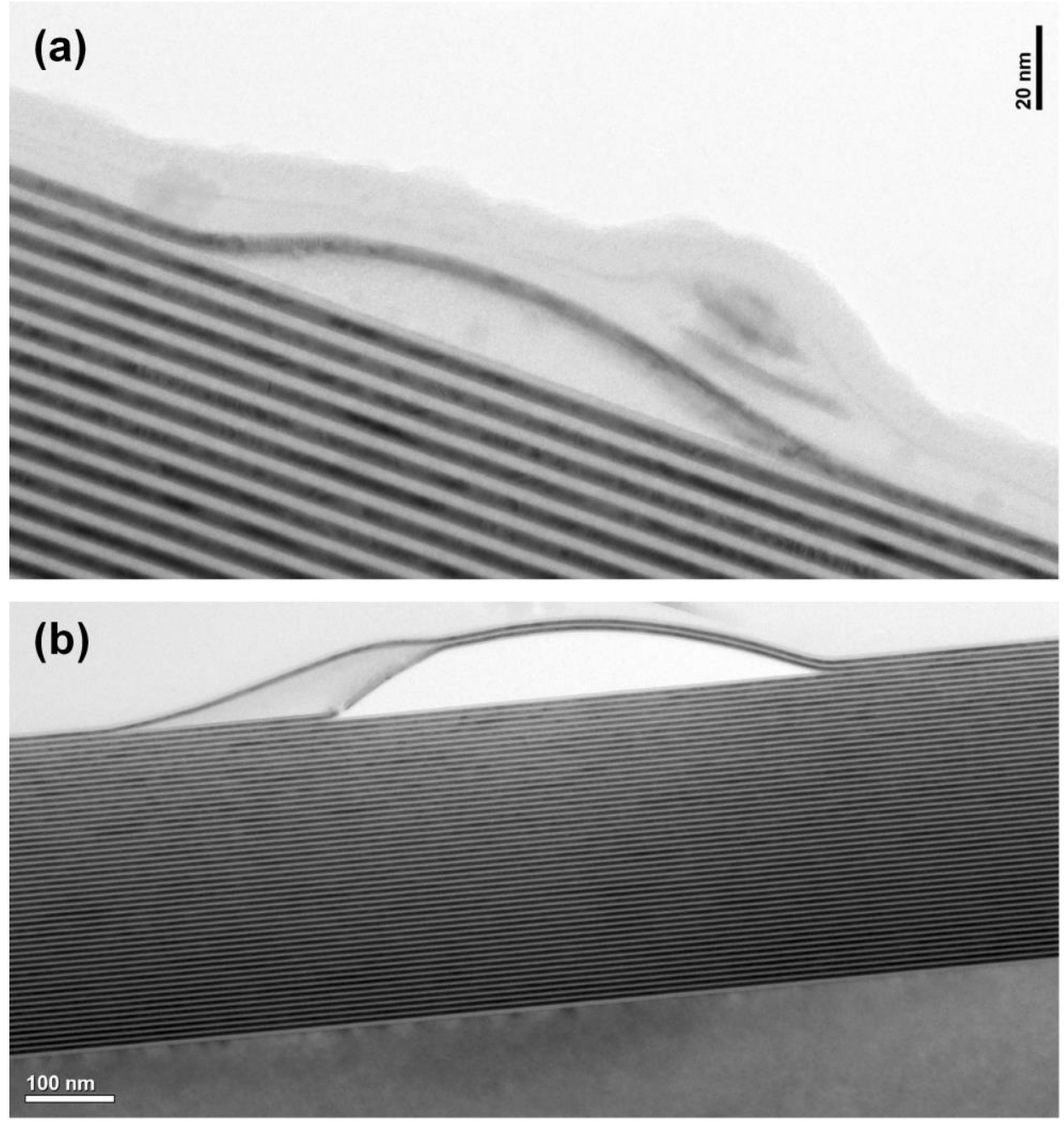

Figure 3: TEM cross-sections from a D-exposed sample. Si layers appear light and Mo layers dark. (a) Single blister with delamination occurring at the outermost Mo-on-Si interface. (b) Double-blister structure with overlapping delaminations occurring at the outermost two Mo-onSi interfaces. 

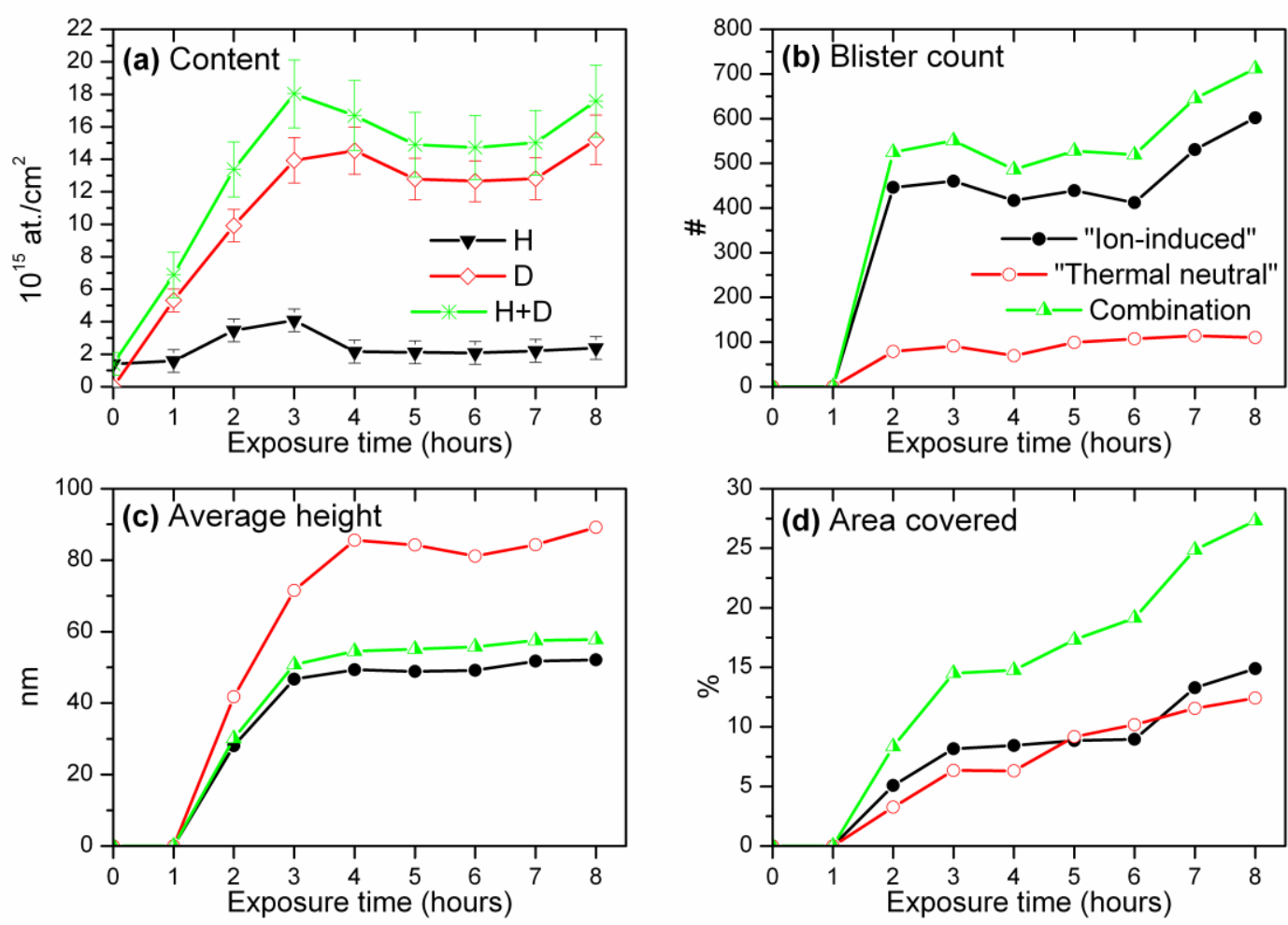

Figure 4: (a) $\mathrm{H}$ - and D-contents in the outermost $30 \mathrm{~nm}$ of the ML sample as a function of exposure time, as determined by ERD. (b-d) Analysis of resultant blisters distributions derived from $25 \times 25 \mu \mathrm{m}^{2}$ AFM scans: (b) blister count; (c) average blister height; and (d) percentage surface area covered. The trace labels shown on panel (b) apply to panels (b-d). 


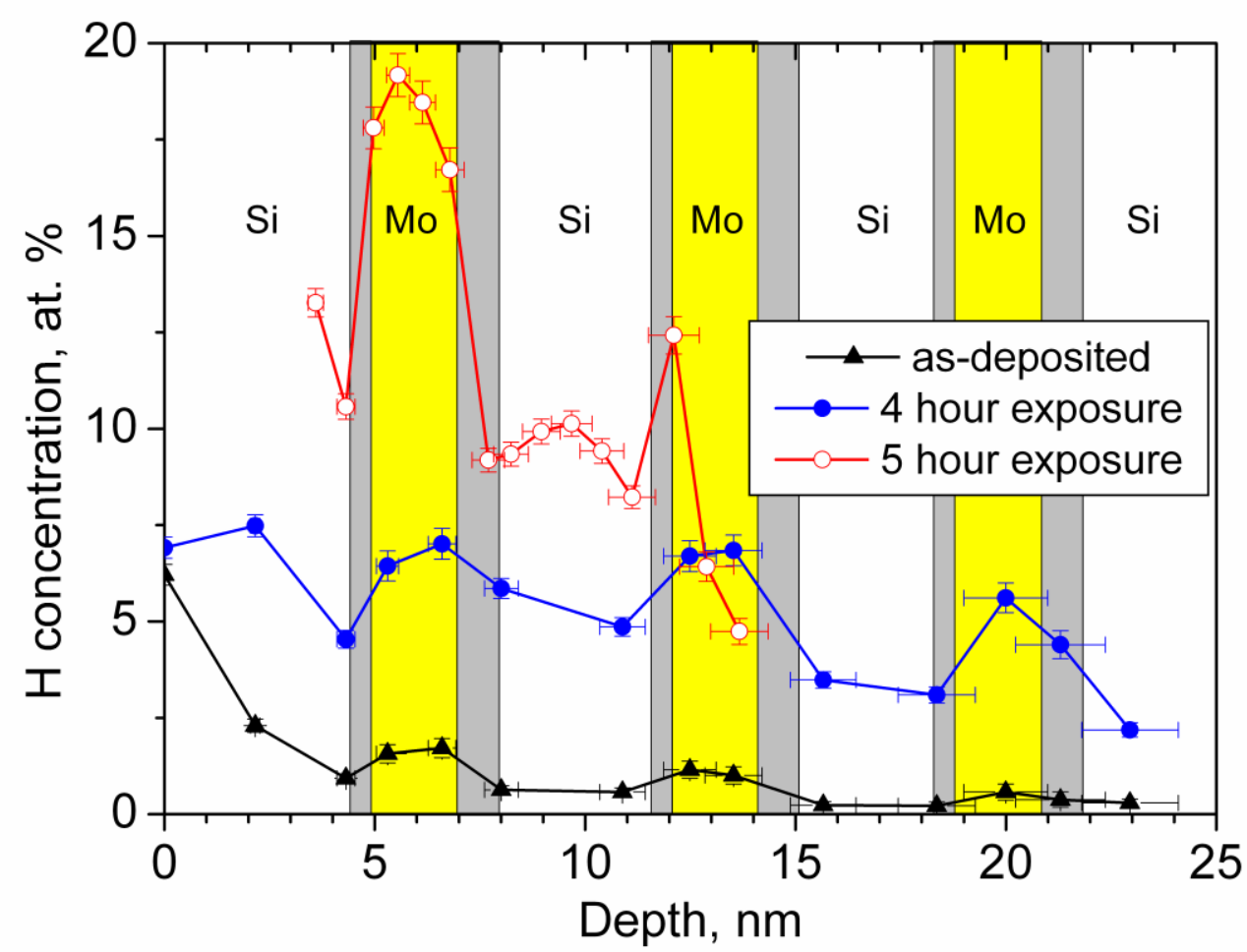

Figure 5: H concentration depth profiles measured by NRA. The approximate locations of individual Si and Mo layers and the associated interface mixing zones are indicated relative to the sample surface. 